Research Paper

\title{
Timing of mTORI usage and outcomes in kidney transplant recipients
}

\author{
Lee-Moay Lim, M.D.1,2,3, Lan-Fang Kung4, Mei-Chuan Kuo, M.D.1,5, A-Mei Huang,,6,7, Hung-Tien Kuo, \\ M.D. ${ }^{1,5 凶}$ \\ 1. Division of Nephrology, Department of Internal Medicine, Kaohsiung Medical University Hospital, Kaohsiung Medical University, Kaohsiung, Taiwan. \\ 2. Graduate Institute of Clinical Medicine, College of Medicine, Kaohsiung Medical University, Kaohsiung, Taiwan. \\ 3. Faculty of Medicine, College of Medicine, Kaohsiung Medical University, Kaohsiung, Taiwan. \\ 4. Department of Nursing, Kaohsiung Medical University Hospital, Kaohsiung, Taiwan. \\ 5. Faculty of Renal Care, College of Medicine, Kaohsiung Medical University, Kaohsiung, Taiwan. \\ 6. Graduate Institute of Medicine, College of Medicine, Kaohsiung Medical University, Kaohsiung, Taiwan. \\ 7. Department of Biochemistry, School of Medicine, College of Medicine, Kaohsiung Medical University, Kaohsiung, Taiwan.
}

$\square$ Corresponding author: Hung-Tien Kuo M.D., Division of Nephrology, Department of Internal Medicine, Kaohsiung Medical University Hospital, Kaohsiung Medical University, No.100, Tzyou First Road, Kaohsiung 807, Taiwan. TEL: 8867 3121101, FAX: 8867 3122810, E-mail: hutiku@kmu.edu.tw.

(c) The author(s). This is an open access article distributed under the terms of the Creative Commons Attribution License (https://creativecommons.org/licenses/by/4.0/). See http://ivyspring.com/terms for full terms and conditions.

Received: 2020.09.24; Accepted: 2020.12.18; Published: 2021.01.09

\begin{abstract}
The introduction of mammalian target of rapamycin inhibitors (mTORi) as immunosuppressive agents has changed the landscape of calcineurin inhibitor-based immunosuppressive regimens. However, the timing of mTORi conversion and its associated outcomes in kidney transplantation have conflicting results. This study investigated the effect of early or late mTORi post-transplant initiation on major transplant outcomes, including post-transplant malignancy, in kidney transplant recipients in our center. We enrolled 201 kidney transplant recipients with surviving function grafts of $>3$ months between 1983 and 2016 . Patients were divided into three groups: early mTORi (initiated within 6 months of kidney transplantation), late mTORi, (mTORi initiation $>6$ months after kidney transplantation) and no mTORi. The mean creatinine at conversion was $1.46 \pm 0.48 \mathrm{mg} / \mathrm{dL}$ and $1.30 \pm 0.53 \mathrm{mg} / \mathrm{dL}$ for the early and late mTORi groups, respectively. During the study period, $10.5 \%$ of mTORi users and $19.2 \%$ of mTORi nonusers developed malignancy, mainly urothelial carcinoma. After adjustment for confounding factors, mTORi users were found to have a lower incidence of post-transplant malignancy than did nonusers (adjusted OR: 0.28, $P=0.04$ ). No significant difference was observed between early and late mTORi users. Our results verified the potential advantages of $m$ TORi usage in reducing cancer incidence after kidney transplantation. However, no significant result was found related to the timing of $\mathrm{mTORi}$ introduction. Future studies should include a longer observation period with a larger cohort.
\end{abstract}

Key words: kidney transplantation; immunosuppressant; mammalian target of rapamycin inhibitor (mTORI); outcomes; malignancy

\section{Introduction}

Advancements in surgical and medical techniques and immunosuppressive agents have led to remarkable short-term outcomes in kidney transplantation. However, with improved patient survival and an aging transplant population, longterm complications have become a major challenge [1]. The prolonged modification of the immune system of kidney transplant recipients is associated with the risk of opportunistic infection and various cancers [1].

The incidence of malignancy in kidney transplant recipients is at least 2-4-fold higher than that in the age- and sex-matched general population $[1,2]$, with higher cancer-related mortality [3]. The pattern of post-transplant malignancy (PTM) varies among geographical regions. Studies from Western countries have indicated that non-melanoma skin cancer is the most common PTM [4]. By contrast, skin cancer has a lower incidence in the Asian population. In Japan, gastric and kidney cancer are the two most frequent PTMs, whereas in Korea, they are gastric cancer and lymphoma [5, 6]. In Hong Kong, nonHodgkin lymphoma has the highest standardized incidence ratio among PTMs [7], and in Taiwan and 
China, urothelial carcinoma is the most common PTM (approximately 40\%) [8, 9].

Immunosuppressive therapies may directly affect cancer growth through various mechanisms. Cyclosporin and tacrolimus increase the risk of malignancy in kidney transplant recipients [10, 11], whereas mammalian target of rapamycin inhibitors (mTORi) reduce the risk $[12,13]$. However, mTORi use in kidney transplantation is associated with inferior graft survival $[14,15]$ and increased mortality risk $[16,17]$. In recent meta-analysis, Wolf $S$. et al found that initiation of mTORi within 3 months of kidney transplantation may reduce the future risk of malignancy [18] but in Hahn et al. analysis, mTORi treatment failed to demonstrate a reduction in cancer risk [19]. These controversial results regarding the role of mTORi and timing of introduction in transplant outcomes need further evidence for verification.

This study investigated the effect of early and late mTORi initiation on major transplant outcomes, including PTMs, in kidney transplant recipients.

\section{Materials and Methods}

\section{Study population}

This retrospective observational study included kidney transplant recipients followed up in a medical center in southern Taiwan from January 1983 to April 2016. The immunosuppressive treatment in our hospital consisted of induction therapy (methylprednisolone and basiliximab, an interleukin-2 receptor antagonist) and maintenance treatment (based on tacrolimus, mycophenolate mofetil, and prednisolone). $\mathrm{mTORi}$, including rapamycin and everolimus, were included in the maintenance regimen if the graft function was stable 3 months after transplantation, minimizing the calcineurin inhibitor (CNI) dosage. Inclusion criteria were kidney transplant recipients who had mTORi as one of their maintenance immunosuppressive agents with a treatment duration of $>3$ months. We excluded patients who were diagnosed with malignancy before the introduction of mTORi. A total of 201 kidney transplant recipients who survived with functioning grafts for $>3$ months were included.

At the baseline visit, the patients' sociodemographic characteristics, medical history, and current maintenance immunosuppressive medications were recorded. Their medical histories were confirmed through doctors' chart reviews. The patients' biochemistry measurements were collected at the baseline visit, before and after kidney transplantation, and at the beginning of mTORi treatment. The patients were stratified into three groups according to mTORi usage and time of initiation: no mTORi, early mTORi (mTORi initiation within 6 months of kidney transplantation), and late mTORi (mTORi initiation $>6$ months after kidney transplantation). Our patients received regular follow-up in the nephrology and urology clinics after the transplant surgery for immune status and disease progression monitoring. Screening for malignancy, including tumor marker survey, kidney and liver sonography, chest $\mathrm{X}$-ray, and urine cytology, was conducted every 6 months.

\section{Ethics statement}

The study protocol was approved by the Institutional Review Board of Kaohsiung Medical University Hospital (KMUHIRB-E(II)-20150089). Written informed consent was obtained from all patients, and all clinical investigations were conducted according to the principles expressed in the Declaration of Helsinki. The patients provided consent for the publication of clinical details.

\section{Outcomes}

The association of early and late mTORi initiation with major transplant outcomes in kidney transplant recipients was analyzed, which included overall survival, patient survival, first-year rejection, and PTMs.

\section{Statistical analysis}

The baseline characteristics of all the patients are expressed as percentages for categorical data and means \pm standard deviations for normally distributed continuous variables. Multivariate logistic regression analyses were performed to evaluate the association among mTORi, rejection, and PTM. Multivariate Cox regression analysis was conducted to evaluate the association between mTORi and graft failure and mortality. $P<0.05$ was considered statistically significant. All analyses were performed using STATA statistical software, v11 (StataCorp LP, College Station, TX, USA). Graft and patient survival estimates were compared using the log-rank test. Kaplan-Meier estimates of graft/patient survivals were compared using the log-rank test. Hazard ratios for graft failure/mortality were calculated using the multivariate Cox regression analysis. Odds ratios for rejection/malignancy were examined using multivariate logistic regression analysis. Adjustments were made for the following major recipient factors: age, body mass index, diabetes mellitus, cause of end-stage renal disease, hepatitis B virus (HBV), hepatitis $\mathrm{C}$ virus (HCV), and immunosuppression. 


\section{Results}

The baseline characteristics and demographic data of the study cohort are presented in Table 1. A total of 201 kidney transplant recipients were included, with a mean age of $46.2 \pm 11.6$ years and body mass index of $22.3 \pm 3.7 \mathrm{~kg} / \mathrm{m}^{2}$. Of the included patients, $51.7 \%$ were men, and $11.6 \%$ and $7 \%$ were $\mathrm{HBV}$ and HCV carriers, respectively. The mean follow-up duration was $2368 \pm 1700$ days. The mean duration of mTORi use was $821.3 \pm 3225$ days (Table $1)$.

Table 1. Baseline Characteristic and Demographic Data of the mTORI User and Non-users

\begin{tabular}{lllll}
\hline & $\begin{array}{l}\text { No mTORI } \\
(\mathrm{N}=78)\end{array}$ & $\begin{array}{l}\text { Early mTORI } \\
(\mathrm{N}=39)\end{array}$ & $\begin{array}{l}\text { Late mTORI } \\
(\mathrm{N}=84)\end{array}$ & $\begin{array}{l}\text { Total } \\
(\mathrm{N}=201)\end{array}$ \\
\hline Age at Tx, years & $46.8 \pm 10.7$ & $45.8 \pm 12.4$ & $45.8 \pm 12.1$ & $46.2 \pm 11.6$ \\
Male, $\%$ & $46.2 \%$ & $48.7 \%$ & $58.3 \%$ & $51.7 \%$ \\
BMI, Kg/m² & $21.6 \pm 2.8$ & $22.3 \pm 4.3$ & $22.9 \pm 4.1$ & $22.3 \pm 3.7$ \\
Initial Cr, mg/dl & - & $1.46 \pm 0.48$ & $1.30 \pm 0.53$ & - \\
HBV & $9(12.7 \%)$ & $3(7.9 \%)$ & $10(12.5 \%)$ & $22(11.6 \%)$ \\
HCV & $7(10.1 \%)$ & $3(7.9 \%)$ & $3(3.8 \%)$ & $13(7 \%)$ \\
DM & $12(15.4 \%)$ & $12(30.7 \%)$ & $18(21.4 \%)$ & $42(20.9 \%)$ \\
Hypertension & $63(80.8 \%)$ & $30(76.9 \%)$ & $60(71.4 \%)$ & $153(76.1 \%)$ \\
Living donor & $7(9.0 \%)$ & $4(12.3 \%)$ & $3(3.6 \%)$ & $14(7.0 \%)$ \\
Induction & $51(78.5 \%)$ & $31(91.2 \%)$ & $59(88.1 \%)$ & $141(84.9 \%)$ \\
Tacrolimus & $69(89.6 \%)$ & $36(92.3 \%)$ & $64(76.2 \%)$ & $169(84.5 \%)$ \\
MMF & $67(85.9 \%)$ & $39(100 \%)$ & $81(96.4 \%)$ & $187(93.0 \%)$ \\
Steroid-free in 3 m & $6(8 \%)$ & $14(35.9 \%)$ & $14(17.1 \%)$ & $34(16.9 \%)$ \\
Follow-up days & $2420 \pm 1993$ & $1643 \pm 1072$ & $2656 \pm 1555$ & $2368 \pm 1700$ \\
Overall graft & $27(34.6 \%)$ & $7(18 \%)$ & $18(21.4 \%)$ & $52(25.9 \%)$ \\
failure & & & & \\
Back to dialysis & $17(21.8 \%)$ & $4(10.3 \%)$ & $11(13.1 \%)$ & $32(15.9 \%)$ \\
Mortality & $11(14.1 \%)$ & $3(7.7 \%)$ & $9(10.7 \%)$ & $23(11.4 \%)$ \\
Rejection in 1 yr & $5(6.4 \%)$ & $5(12.8 \%)$ & $10(11.9 \%)$ & $20(10.0 \%)$ \\
Post-Tx & $15(19.2 \%)$ & $5(12.8 \%)$ & $8(9.5 \%)$ & $28(13.9 \%)$ \\
Malignancy & & & & \\
\hline $\begin{array}{l}\text { Abbreviations: Tx: transplant; BMI, body mass index; Cr, creatinine; HBV, hepatitis } \\
\text { B; HCV, hepatitis C; DM, diabetes mellitus; MMF, mycophenolate }\end{array}$ & mofetil; m, \\
month; yr, year. & & & &
\end{tabular}

The mean creatinine level at conversion was 1.46 $\pm 0.48 \mathrm{mg} / \mathrm{dL}$ and $1.30 \pm 0.53 \mathrm{mg} / \mathrm{dL}$ for the early and late mTORi groups, respectively (Table 1). Most patients received the interleukin-2 receptor antagonist Simulect ${ }^{\circledR}$ (Novartis Pharma Stein AG, Switzerland) as the induction agent $(84.9 \%)$. The maintenance regimens consisted mostly of tacrolimus $(84.5 \%)$ and mycophenolate mofetil (MMF, 93.0\%). Of the included patients, $16.9 \%$ had steroid-free maintenance treatment for 3 months after transplantation (Table 1). The overall mortality rate was $11.4 \%$, mainly due to cardiovascular events, followed by PTM and infection (Table 2).

Of the 201 patients, 28 (13.9\%) developed PTM, 15 of whom (19.2\%) were mTORi nonusers. The most common PTM was urothelial carcinoma $(\mathrm{n}=10)$, followed by hepatocellular carcinoma $(n=7)$ (Table 3).
Table 2. Causes of Death between mTORI users and Non-users

\begin{tabular}{lllll}
\hline & $\begin{array}{l}\text { No mTORI } \\
(\mathrm{N}=78)\end{array}$ & $\begin{array}{l}\text { Early mTORI } \\
(\mathrm{N}=39)\end{array}$ & $\begin{array}{l}\text { Late mTORI } \\
(\mathrm{N}=84)\end{array}$ & $\begin{array}{l}\text { Total } \\
(\mathrm{N}=201)\end{array}$ \\
\hline Cardiovascular & 4 & 2 & 3 & 9 \\
Infection & 2 & 0 & 3 & 5 \\
Malignancy & 3 & 1 & 3 & 7 \\
Others & 2 & 0 & 0 & 2 \\
All & 11 & 3 & 9 & 23 \\
\hline
\end{tabular}

Table 3. Types of Malignancy

\begin{tabular}{ll}
\hline Type of malignancy & Frequency \\
\hline Breast cancer & 1 \\
Colon cancer & 1 \\
Cervix cancer & 1 \\
Hepatocellular carcinoma & 7 \\
Lung cancer & 2 \\
Lymphoma & 1 \\
Pancreas cancer & 1 \\
Prostate cancer & 2 \\
Renal cell carcinoma & 1 \\
Urothelial carcinoma & 10 \\
Peritoneal carcinomatosis & 1 \\
Total & 28 \\
\hline
\end{tabular}

We compared overall graft and patient survival with and without mTORi use. In the multivariate analysis, no significant association was noted between these two groups, but the early mTORi group exhibited a trend of better survival outcomes than did the other groups (Figure 1A, 1B). mTORi users had higher incidents of graft rejection 1 year after transplantation, but this association was not significant in multivariate analysis (adjusted OR: 1.40, $P=0.59$; Figure 2). After adjustment for confounding factors, mTORi users had a lower incidence of PTM than did nonusers (adjusted OR: 0.28, $P=0.04$ ), but no significant difference was observed between early and late mTORi users (Figure 3).

\section{Discussion}

In this study, we examined the timing of mTORi usage and its related outcomes and reviewed the incidence of PTM in kidney transplant recipients. mTORi users had a lower incidence of PTM than did nonusers. However, no differences were observed between early and late mTORi users in patient survival, graft survival, or acute rejection.

mTORi exert complex effects on the immune system due to the multiple roles of the mTOR signaling pathway in the immune cascade [20]. mTOR pathway inhibition interferes with cell growth and proliferation and suppresses the immune response by interfering with T-cell proliferation [21, 22]. Compared with CNIs, mTORi have less direct nephrotoxicity. mTORi combined with low-dose cyclosporine lowers skin cancer incidence [23, 24]. In the CONVERT trial, kidney transplant recipients with 
sirolimus and a CNI-free regimen had a lower overall incidence of malignancies ( 2.1 vs 6.0 malignancies per 100 person-years of exposure) [23]. However, in the ZEUS study, which compared everolimus with CNI elimination versus a standard CNI regimen, malignancy was reported in $1.6 \%$ of everolimustreated patients compared with $6.4 \%$ in the standard CNI group [25]. These results imply that mTORi provide protection against PTM.

Compared with the general population, kidney transplant recipients have a higher risk of cancer, especially skin cancer and virus-related cancers [1, 29]. Cancers with higher standardized incidence ratios observed in organ transplant recipients are nonmelanoma skin cancers, followed by Kaposi sarcoma and posttransplant lymphoproliferative disease [26]. Urothelial carcinoma is the most common PTM in Taiwan [27, 28]. The most common PTMs in our study were urothelial carcinoma and

A

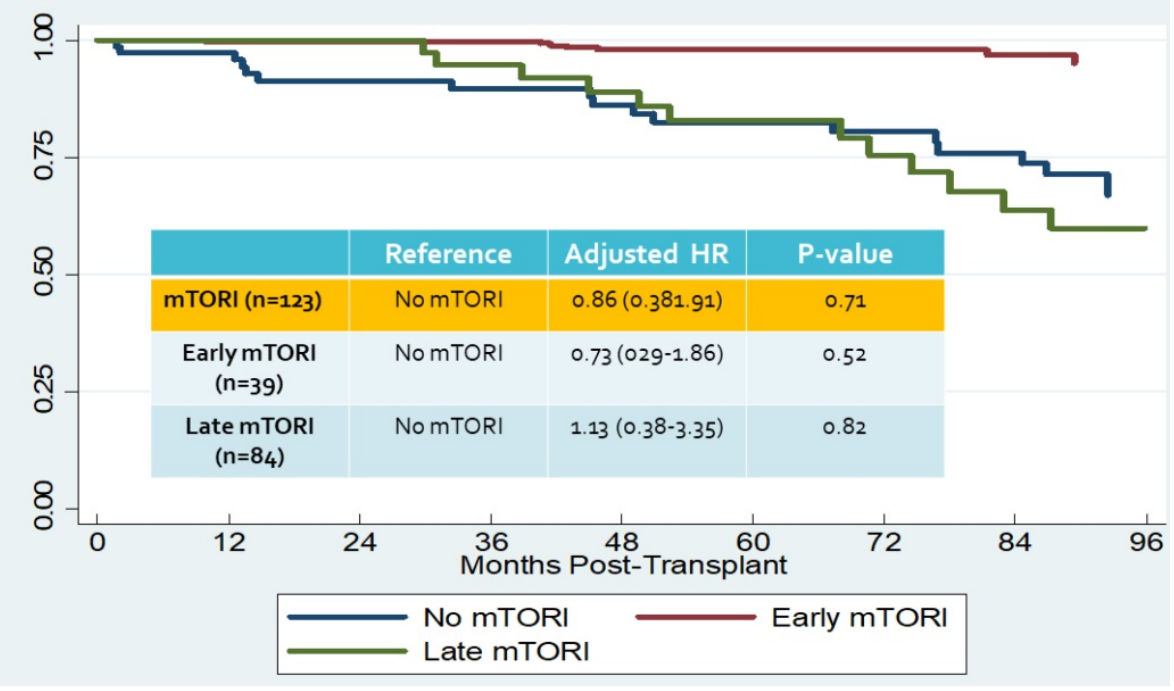

B

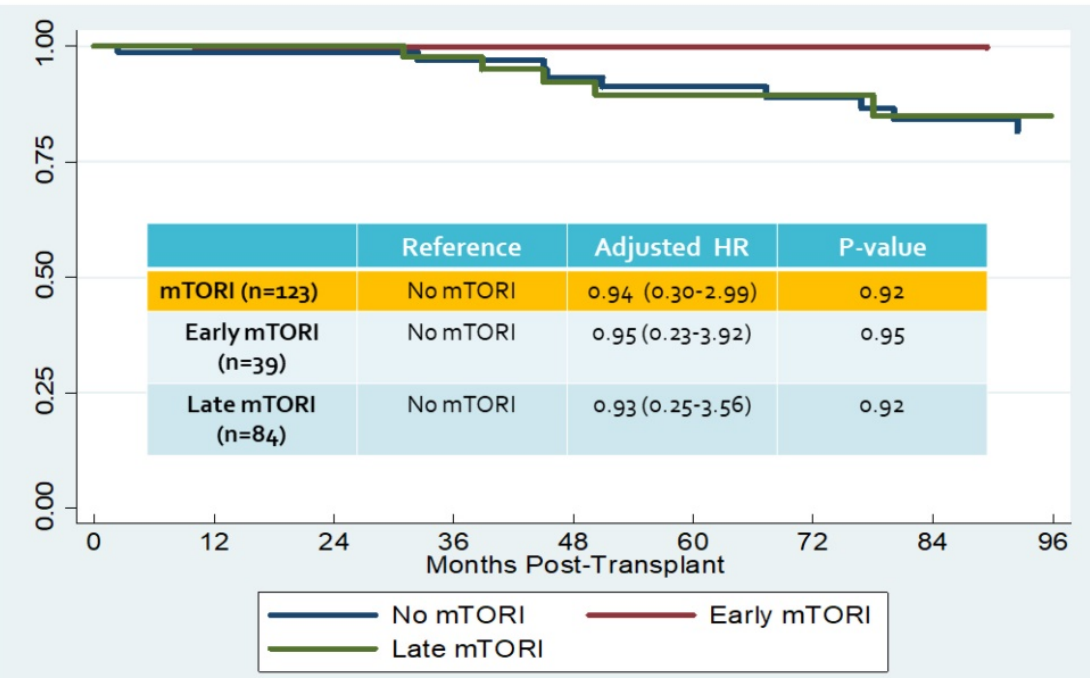

Figure 1. A. Overall graft survival in mTORI User and Non-users. Figure 1B. Patients survival in mTORI User and Non-users. hepatocellular carcinoma. In a nationwide cohort population-based study, Tsai et al. demonstrated that heart, lung, kidney, and liver transplant recipients had a three-fold risk of de novo cancer compared with the general population [29]. Kidney transplant recipients were associated with a standardized incidence ratio of $10.93(95 \% \mathrm{CI}, 9.20-12.99)$ for urinary tract malignancies, among which bladder cancer was the most common [29]. The high prevalence of HBV and HCV infection in Taiwan may explain the increased risk of hepatocellular carcinoma in organ transplant recipients, but this association requires further exploration by comparing kidney transplant recipients with and without $\mathrm{HBV}$ and $\mathrm{HCV}$.

The mTORi-based immunosuppressive regimens were introduced in CNI minimization/ withdrawal strategies to prevent CNI-associated nephrotoxicity. Early conversion of CNI to mTORi within the first 6 months of transplantation has been proposed as a potential strategy to reduce $\mathrm{CNI}$ toxicity [30]. However, an increased risk of graft loss was observed in mTORitreated patients. In the ZEUS study, patients were randomized to everolimus or cyclosporine arms at 4.5 months post-transplant, and a higher biopsy-confirmed acute rejection (BCAR) was observed in the everolimus arm [31,32]. In the CONCEPT study in which patients were randomized at 3 months, the BCAR rate was $17 \%$ in the sirolimus arm compared with $8 \%$ in the cyclosporine arm. Most rejection occurred in the sirolimus arm when steroids were discontinued at 8 months after transplantation [33]. To investigate the effect of late conversion of mTORi (>6 months post-transplantation), the CONVERT trial revealed no significant difference in renal benefit, BCAR, graft, and patient survival [34]. In our study, minimization in the CNI dosage was noted in the 
mTORi user group, but this was not significantly associated with patients' survival, graft survival, or acute rejection.

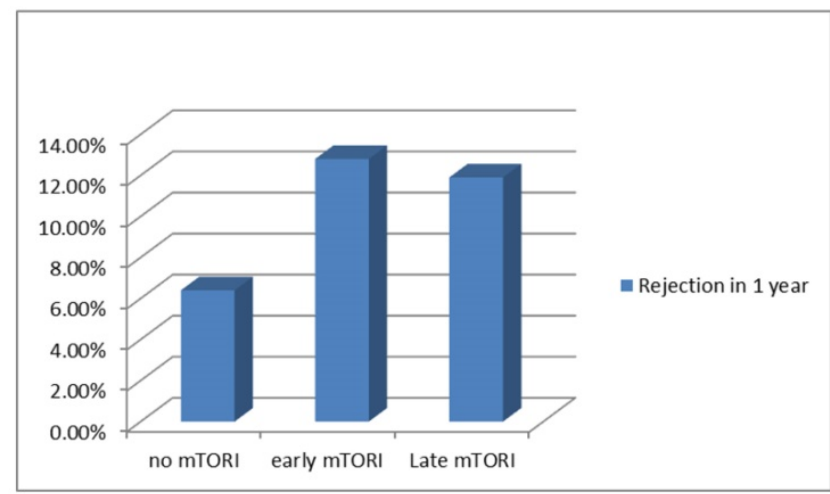

\begin{tabular}{|l|l|l|l|}
\hline & Reference & Adjusted OR & P-value \\
\hline mTORI $(n=123)$ & No mTORI & $1.40(0.41-4.82)$ & 0.59 \\
\hline Early mTORI $(n=39)$ & No mTORI & $1.57(0.38-6.45)$ & 0.52 \\
\hline Late mTORI $(n=84)$ & No mTORI & $1.25(0.30-5.23)$ & 0.76 \\
\hline
\end{tabular}

Figure 2. Rejection Rate in 1 year of mTORI User and Non-users.

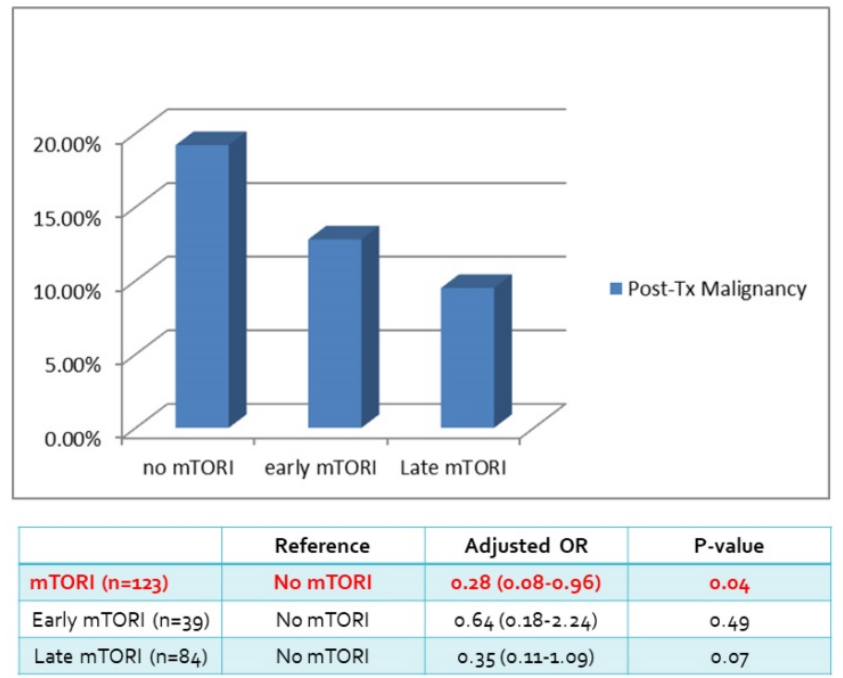

Figure 3. Post-transplant Malignancy rate in in mTORI User and Non-users.

This study has several limitations. First, it was a single-center observational study with a small cohort. Second, the follow-up period was short, which might not reflect longer-term graft outcomes and PTM incidence. Third, sirolimus and everolimus were studied as a class (mTORi) due to the small number of everolimus used. Finally, the modification of immunosuppressive agents in kidney transplant recipients might affect this study's ability to correlate the effect of the dose effect of immunosuppressive agents on PTM incidence.

\section{Conclusion}

In our cohort of renal transplant recipients, mTORi use was associated with a decreased risk of
PTM. However, no difference in mortality, graft loss, and PTM was noted in patients with early conversion to mTORi (within 6 months of kidney transplantation). The most common PTMs were urothelial and hepatocellular carcinoma. The discrepancy in cancer occurrence highlighted the need for developing specific strategies for cancer surveillance in kidney transplant recipients.

\section{Acknowledgments}

The authors thank the entire chronic kidney disease care team at Kaohsiung Medical University Hospital for assistance in sample collection.

\section{Support and Financial Disclosure Declaration}

This study was supported by the Research Program funded by the Kaohsiung Medical University Hospital (KMUH104-4M09 and KMUH105-5M12.).

\section{Competing Interests}

The authors have declared that no competing interest exists.

\section{References}

1. Engels EA, Pfeiffer RM, Fraumeni JF, Jr., Kasiske BL, Israni AK, Snyder JJ, et al. Spectrum of cancer risk among US solid organ transplant recipients. JAMA. 2011;306(17):1891-901.

2. Grulich AE, van Leeuwen MT, Falster MO, Vajdic CM. Incidence of cancers in people with HIV/AIDS compared with immunosuppressed transplant recipients: a meta-analysis. Lancet. 2007;370(9581):59-67.

3. Kiberd BA, Rose C, Gill JS. Cancer mortality in kidney transplantation. Am J Transplant. 2009;9(8):1868-75.

4. Agraharkar ML, Cinclair RD, Kuo YF, Daller JA, Shahinian VB. Risk of malignancy with long-term immunosuppression in renal transplant recipients. Kidney Int. 2004;66(1):383-9.

5. Hoshida Y, Tsukuma H, Yasunaga Y, Xu N, Fujita MQ, Satoh T, et al. Cancer risk after renal transplantation in Japan. Int J Cancer. 1997;71(4):517-20.

6. Hwang JK, Moon IS, Kim JI. Malignancies after kidney transplantation: a 40-year single-center experience in Korea. Transpl Int. 2011;24(7):716-21.

7. Cheung CY, Lam MF, Chu KH, Chow KM, Tsang KY, Yuen SK, et al. Malignancies after kidney transplantation: Hong Kong renal registry. Am J Transplant. 2012;12(11):3039-46.

8. Xiao J, Zhu X, Hao GY, Zhu YC, Hou HJ, Zhang J, et al. Association between urothelial carcinoma after kidney transplantation and aristolochic acid exposure: the potential role of aristolochic acid in HRas and TP53 gene mutations. Transplant Proc. 2011;43(10):3751-4.

9. Wang LJ, Wong YC, Huang CC. Urothelial carcinoma of the native ureter in a kidney transplant recipient. J Urol. 2010;184(2):728.

10. Maluccio M, Sharma V, Lagman M, Vyas S, Yang H, Li B, et al. tacrolimus enhances transforming growth factor-betal expression and promotes tumor progression. Transplantation. 2003;76(3):597-602.

11. Herman $M$, Weinstein $T$, Korzets A, Chagnac A, Ori $Y$, Zevin D, et al. Effect of cyclosporin A on DNA repair and cancer incidence in kidney transplant recipients. J Lab Clin Med. 2001;137(1):14-20.

12. Lim WH, Eris J, Kanellis J, Pussell B, Wiid Z, Witcombe D, et al A systematic review of conversion from calcineurin inhibitor to mammalian target of rapamycin inhibitors for maintenance immunosuppression in kidney transplant recipients. Am J Transplant. 2014;14(9):2106-19.

13. Campbell SB, Walker R, Tai SS, Jiang $Q$, Russ GR. Randomized controlled trial of sirolimus for renal transplant recipients at high risk for nonmelanoma skin cancer. Am J Transplant. 2012;12(5):1146-56.

14. Xie X, Jiang $Y$, Lai X, Xiang S, Shou Z, Chen J. mTOR inhibitor versus mycophenolic acid as the primary immunosuppression regime combined with calcineurin inhibitor for kidney transplant recipients: a meta-analysis. BMC Nephrol. 2015;16:91.

15. Ekberg H, Bernasconi C, Tedesco-Silva H, Vitko S, Hugo C, Demirbas A, et al. Calcineurin inhibitor minimization in the Symphony study: observational results 3 years after transplantation. Am J Transplant. 2009;9(8):1876-85.

16. Srinivas TR, Schold JD, Guerra G, Eagan A, Bucci CM, Meier-Kriesche HU. Mycophenolate mofetil/sirolimus compared to other common 
immunosuppressive regimens in kidney transplantation. Am J Transplant. 2007;7(3):586-94

17. Isakova $\mathrm{T}$, Xie H, Messinger S, Cortazar F, Scialla JJ, Guerra G, et al. Inhibitors of mTOR and risks of allograft failure and mortality in kidney transplantation. Am J Transplant. 2013;13(1):100-10.

18. Wolf S, Hoffmann VS, Habicht A, Kauke T, Bucher J, Schoenberg M, et al. Effects of mTOR-Is on malignancy and survival following renal transplantation: A systemic review and meta-analysis of randomized trials with a minimum follow-up of 24 months. PLoS One 2018 Apr16;13(4):e0194975.

19. Hahn D, Hodson EM, Hamiwka LA, Lee VW, Chapman JR, Craig JC, et al. Target of rapamycin inhibitors (TOR-I; sirolimus and everolimus) for primary immunosuppression in kidney transplant recipients. Cochrane Database Syst Rev. 2019;12:CD004290.

20. Cobbold SP. The mTOR pathway and integrating immune regulation. Immunology. 2013;140(4):391-8.

21. Lee RA, Gabardi S. Current trends in immunosuppressive therapies for renal transplant recipients. Am J Health Syst Pharm. 2012;69(22):1961-75.

22. Halloran PF. Immunosuppressive drugs for kidney transplantation. N Engl J Med. 2004;351(26):2715-29.

23. Alberu J, Pascoe MD, Campistol JM, Schena FP, Rial Mdel C, Polinsky M, et al. Lower malignancy rates in renal allograft recipients converted to sirolimus-based, calcineurin inhibitor-free immunotherapy: 24-month results from the CONVERT trial. Transplantation. 2011;92(3):303-10.

24. Campistol JM, Eris J, Oberbauer R, Friend P, Hutchison B, Morales JM, et al. Sirolimus therapy after early cyclosporine withdrawal reduces the risk for cancer in adult renal transplantation. J Am Soc Nephrol. 2006;17(2):581-9.

25. Luan FL, Hojo M, Maluccio M, Yamaji K, Suthanthiran M. Rapamycin blocks tumor progression: unlinking immunosuppression from antitumor efficacy. Transplantation. 2002;73(10):1565-72.

26. Vajdic CM, van Leeuwen MT. Cancer incidence and risk factors after solid organ transplantation. Int J Cancer. 2009;125(8):1747-54

27. Li WH, Chen YJ, Tseng WC, Lin MW, Chen TJ, Chu SY, et al. Malignancies after renal transplantation in Taiwan: a nationwide population-based study. Nephrol Dial Transplant. 2012;27(2):833-9.

28. Lee KF, Tsai YT, Lin CY, Hsieh CB, Wu ST, Ke HY, et al. Cancer Incidence among Heart, Kidney, and Liver Transplant Recipients in Taiwan. PLoS One. 2016;11(5):e0155602

29. Tsai HI, Lee CW, Kuo CF, See LC, Liu FC, Chiou MJ, et al. De novo malignancy in organ transplant recipients in Taiwan: a nationwide cohort population study. Oncotarget. 2017;8(22):36685-95.

30. de Fijter JW, Holdaas H, Øen O, Sanders J-S, Sundar S, Bemelman FJ, et al. Early Conversion From Calcineurin Inhibitor- to Everolimus-Based Therapy Following Kidney Transplantation: Results of the Randomized ELEVATE Trial. Am J Transplant. 2017 Jul;17(7):1853-1867.

31. Budde K, Lehner F, Sommerer C, Arns W, Reinke P, Eisenberger U, et al. Conversion from cyclosporine to everolimus at 4.5 months posttransplant: 3-year results from the randomized ZEUS study. Am J Transplant. 2012;12(6):1528-40.

32. Sommerer C, Budde K, Zeier M, Wuthrich RP, Reinke P, Eisenberger U, et al. Early conversion from cyclosporine to everolimus following living-donor kidney transplantation: outcomes at 5 years posttransplant in the randomized ZEUS trial. Clin Nephrol. 2016;85(4):215-25.

33. Lebranchu Y, Thierry A, Toupance O, Westeel PF, Etienne I, Thervet E, et al. Efficacy on renal function of early conversion from cyclosporine to sirolimus 3 months after renal transplantation: concept study. Am J Transplant. 2009;9(5):1115-23.

34. Schena FP, Pascoe MD, Alberu J, del Carmen Rial M, Oberbauer R, Brennan $\mathrm{DC}$, et al. Conversion from calcineurin inhibitors to sirolimus maintenance therapy in renal allograft recipients: 24 -month efficacy and safety results from the CONVERT trial. Transplantation. 2009;87(2):233-42. 